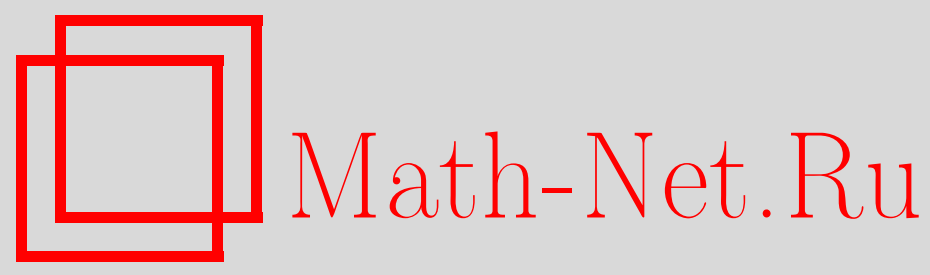

Н. Г. Мощевитин, Об образе декартового прозведения канторовых $\tau$-множеств при гладком отображении, Матем. заметки, 2000, том 68, выпуск 6, 948-950

DOI: https://doi.org/10.4213/mzm1020

Использование Общероссийского математического портала Math-Net.Ru подразумевает, что вы прочитали и согласны с пользовательским соглашением http://www.mathnet.ru/rus/agreement

Параметры загрузки:

IP : 34.229 .45 .116

26 апреля 2023 г., 12:59:28

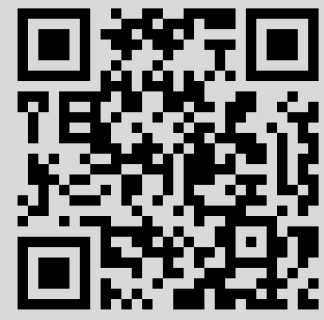




\section{ОБ ОБРАЗЕ ДЕКАРТОВОГО ПРОЗВЕДЕНИЯ КАНТОРОВЫХ $\tau$-МНОЖЕСТВ ПРИ ГЛАДКОМ ОТОБРАЖЕНИИ}

\section{Н. Г. Мощевитин}

Мы будем пользоваться следуюшим определением $\tau$-множества на вещественой прямой.

ОПРЕДЕЛЕНИЕ. Пусть $\mathscr{I} \subset \mathbb{R}$ - некоторый отрезок. Множество $\mathscr{F} \subset \mathscr{I}$ называется $\tau$-множеством $(\tau \geqslant 0)$, если оно может быть получено из отрезка $\mathscr{I}$ последовательным выкидыванием счетного (или конечного) набора интервалов $\Delta_{\nu} \subset \mathscr{I}, \nu=1,2, \ldots$, так, что каждый раз интервал $\Delta_{\nu}$ выкидьвается из некоторого отрезка $M_{\nu} \subset \mathscr{I}$, разбивая его таким образом, что

$$
M_{\nu}=N_{1} \sqcup \Delta_{\nu} \sqcup N_{2} \quad \text { и } \min \left\{\left|N_{1}\right|,\left|N_{2}\right|\right\} \geqslant \tau\left|\Delta_{\nu}\right|
$$

(через $|\cdot|$ здесь и в дальнейшем обозначается длина отрезка или интервала).

Пусть

$$
\mathscr{F}_{j}=\mathscr{I}_{j} \backslash \bigcup_{\nu=1}^{\infty} \Delta_{\nu}^{j}, \quad j=1, \ldots, d,
$$

суть $\tau_{j}$-множества. Положим $I_{j}=\left|\mathscr{I}_{j}\right|$ и $\mathfrak{I}=\mathscr{I}_{1} \times \cdots \times \mathscr{I}_{d}$.

Рассмотрим теперь вещественнозначную функцию $G\left(x_{1}, \ldots, x_{d}\right): \mathfrak{I} \rightarrow \mathbb{R}$ класса $G \in C^{1}(\mathfrak{I})$ и определим величины

$$
\alpha_{j}=\min _{\mathfrak{I}}\left|\frac{\partial G}{\partial x_{j}}\right|, \quad \omega_{j}=\max _{\mathfrak{I}}\left|\frac{\partial G}{\partial x_{j}}\right|, \quad \lambda_{j}=\frac{\omega_{j}}{\alpha_{j}}, \quad j=1, \ldots, d .
$$

Всюду в этой работе мы будем предполагать, что выполнено условие $\alpha_{j}>0, j=1, \ldots, d$, т.е. мы будем требовать “монотонность” функции $G$.

Для неотрицательных чисел $\tau_{1}, \ldots, \tau_{d} \geqslant 0$ будем рассматривать соотношение

$$
\frac{\tau_{1}}{\lambda_{1}+\tau_{1}}+\cdots+\frac{\tau_{d}}{\lambda_{d}+\tau_{d}} \geqslant 1
$$

Нам понадобятся также следующие ниже соотношения, включающие в себя помимо величин $\tau_{j}$ положительные величины $I_{j}$. Пусть $\sigma=\left(\sigma_{1}, \ldots, \sigma_{d}\right)$ - произвольная перестановка чисел $1, \ldots, d$, a $s$-некоторое натуральное число между 1 и $d-1$. Нужные нам соотношения имеют вид

$$
\sum_{j=1}^{s} I_{\sigma_{j}} \geqslant I_{\sigma_{d}}\left[\frac{\omega_{\sigma_{d}}}{1+2 \tau_{\sigma_{d}}}-\left(\omega_{\sigma_{d}}+\alpha_{\sigma_{d}} \tau_{\sigma_{d}}\right) \sum_{j=s+1}^{d-1} \frac{\tau_{\sigma_{j}}}{\lambda_{\sigma_{j}}+\tau_{\sigma_{j}}}\right]
$$

(в случае $s=d-1$ формально $\sum_{j=d}^{d-1}=0$ ).

ТеОРема. Пусть параметры $\tau_{1}, \ldots, \tau_{d}$ удовлетворяют условию (1) $и$, кроме того, выполнены "начальные условия" (2). Тогда для образа множества $\mathscr{F}_{1} \times \cdots \times \mathscr{F}_{d}$ при отображснии $G$ выполнено $G\left(\mathscr{F}_{1}, \ldots, \mathscr{F}_{d}\right)=G\left(\mathscr{I}_{1}, \ldots, \mathscr{I}_{d}\right)$.

ЗАмеч Ание 1 . При $s=2$ и $G\left(x_{1}, x_{2}\right)=x_{1}+x_{2}$ условие (1) можно переписать в виде $\tau_{1} \tau_{2} \geqslant 1$, так что наша теорема в двумерном случае повторяет результат Ньюхауза [1]-[3], являющийся обобщением классического результата Холла [4]; с другой стороны, в этом случае она усиливает частный результат автора из [5]. Интересные двумерные результаты получены Вильямсом в [6]. Многомерный частный случай нашей теоремы имеется у Главки в [7]. Особо отметим совсем недавние работы Астелса [8], в которой получен аналогичный нашему результат для функции $G\left(x_{1}, \ldots x_{d}\right)=$ $x_{1}+\cdots+x_{d}$ (если в нашей теореме положить $G\left(x_{1}, \ldots, x_{d}\right)=x_{1}+\cdots+x_{d}$, то $\alpha_{j}=\omega_{j}=\lambda_{j}=1$ для всех $j$ и наша теорема преврашается в утверждение 2 теоремы 2.3 из [8]). Отметим также работы [9], [10] и книгу [11]. Обзор родственных задач и обширную библиограффию можно найти в [12].

Работа выполнена при финансовой поддержке Российского фонда фундаментальных исследований, грант № 99-09-0357. 
ЗАмечАниЕ 2 . В качестве пояснения в случае $G\left(x_{1}, \ldots, x_{d}\right)=x_{1}+\cdots+x_{d}$ приведем несколько примеров, когда выполняются "начальные условия" (2):

a) $I_{1}=I_{2}=\cdots=I_{d}$, а $\tau_{j}$ - произвольные положительные числа;

b) $I_{j} \geqslant \kappa_{j, k} I_{k}, j, k=1, \ldots, d$, где $\kappa_{j, k}=\frac{\tau_{j}}{1+\tau_{j}} \cdot \frac{1+\tau_{k}}{1+2 \tau_{k}} \in(0 ; 1)$ и, кроме того, величины $\tau_{j}$ удовлетворяют (1).

ЗАмеч АниЕ 3 . По-видимому, для $G\left(x_{1}, \ldots, x_{d}\right)=x_{1}+\cdots+x_{d}$ начальные условия (2) являются неулучшаемыми в случае, когда $\tau_{j}$ удовлетворяют $р а в е н с т в у$

$$
\frac{\tau_{1}}{1+\tau_{1}}+\cdots+\frac{\tau_{d}}{1+\tau_{d}}=1
$$

Вероятно, что при наличии в (1) строгого неравенства, утверждение нашей теоремы останется выполненым, если заменить "начальные условия" (2) на некоторые более слабые требования.

Доказательство теоремы проводится с помощью неэффетивной конструкции, использовавшейся автором в [5], [13], и некоторых геометрических соображений.

СХЕМА ДОКАЗАТЕЛЬСТВА ТЕОРЕМЫ. Предполагая противное, получаем, что в $d$-мерном пространстве (отнесенном к декартовым координатам $\left.x_{1}, \ldots, x_{d}\right)$ при некотором $\gamma \in\left[\min _{\mathfrak{I}} G, \max _{\mathfrak{I}} G\right]$ компактная $(d-1)$-мерная поверхность $\Sigma=\mathfrak{I} \cap\left\{\left(x_{1}, \ldots, x_{d}\right): G\left(x_{1}, \ldots, x_{d}\right)=\gamma\right\}$ полностью покрывается открытыми множествами вида

$$
\mathbb{R}_{1} \times \cdots \times \mathbb{R}_{j-1} \times \Delta^{j} \times \mathbb{R}_{j+1} \times \cdots \times \mathbb{R}_{d}
$$

где $\mathbb{R}_{k}$ - прямая, соответствующая $k$-му координатному направлению, а $\Delta^{j}$ - один из интервалов, выкидываемых из $\mathscr{I}_{j}$ для получения $\mathscr{F}_{j}$. Из этого покрытия выделяется неприводимое конечное подпокрытие. Каждому направлению $j$ ставится в соответствие свой вес

$$
\varphi_{j}=\omega_{j}+\alpha_{j} \tau_{j}, \quad j=1, \ldots, d,
$$

и из покрытия выделяется полоса (4), для которой значение $\varphi_{\nu} \Delta^{\nu}$ минимально. Если в конечном неприводимом покрытии встечаются полосы всех направлений, то, по аналогии с [5] используя теорему Лагранжа о приращениях и оценивая максимум и минимум соответствующих частных производных, имеем

$$
\left|\Delta^{\nu}\right| \omega_{\nu}>\tau_{1} \alpha_{1}\left|\Delta^{1}\right|+\cdots+\tau_{\nu-1} \alpha_{\nu-1}\left|\Delta^{\nu-1}\right|+\tau_{\nu+1} \alpha_{\nu+1}\left|\Delta^{\nu+1}\right|+\cdots+\tau_{d} \alpha_{d}\left|\Delta^{d}\right| .
$$

(Здесь $\left|\Delta^{\nu}\right|$ - ширина той полосы, на которой достигается минимум, a $\left|\Delta^{j}\right|, j \neq \nu$, определяют минимальные шшрины полос $j$-го направления, участвуюших в рассматриваемом конечном покрытии поверхности $\Sigma$.) Из минимальности имеем $\left|\Delta^{\nu}\right| \varphi_{\nu} \leqslant\left|\Delta^{j}\right| \varphi_{j}$ и с учетом определения весов (5) получаем

$$
\frac{\tau_{1}}{\lambda_{1}+\tau_{1}}+\cdots+\frac{\tau_{d}}{\lambda_{d}+\tau_{d}}<1
$$

что противоречит условию (1). (Выбор весов (5) мы поясним ниже.)

Если же в конечном покрытии не встречаются полосы некоторых направлений, то для этих направлений $j$ в (6) слагаемое $\tau_{j} \alpha_{j}\left|\Delta^{j}\right|$ надо заменить на $I_{j}$. Как легко видеть, теперь получаем противоречие с (2). (В последнем рассуждении мы используем то, что $I_{\nu} \geqslant\left(1+2 \tau_{\nu}\right)\left|\Delta^{\nu}\right|$.) Теорема доказана.

Поясним, почему мы выбираем веса по формулам (5). Положим $t_{j}=\alpha_{j} \tau_{j} / \varphi_{j}$. Для получения противоречия надо, чтобы выполнялись следующие условия:

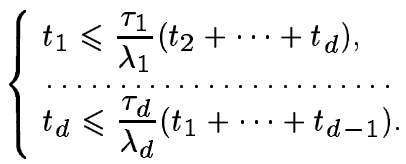


УТВЕРЖДЕНИЕ. Пусть $\tau_{j}$ удовлеторяют (1). Тогда для весов, определенных в (5), условия (7) выполнены.

Покажем, как доказьвать утверждение. Пересечение ортанта $\left\{\left(t_{1}, \ldots, t_{d}\right): t_{j} \geqslant 0, j=1, \ldots\right.$ $\ldots, d\}$ с гиперплоскосью $\pi$, задаваемой уравнением $t_{1}+\cdots+t_{d}=1$, есть правильньй симплекс $\mathfrak{A}$. Множество всех векторов $\left(t_{1}, \ldots, t_{d}\right)$, лежащих в гиперплоскости $\pi$ и удовлетворяющих $(7)$, есть некоторый другой правильный симплекс $\mathfrak{B}$, гомотетичный симплексу $\mathfrak{A}$. Из того, что все $\tau_{j}$ неотрицательны, следует, что центр гомотетии $W$ правильных симплексов $\mathfrak{A}$ и $\mathfrak{B}$ лежит в их пересечении: $W \in \mathfrak{A} \cap \mathfrak{B}$. Остается заметить, что с точностью до пропорциональности $W$ имеет координаты $\tau_{1} /\left(\lambda_{1}+\tau_{1}\right), \ldots, \tau_{d} /\left(\lambda_{d}+\tau_{d}\right)$ и, стало быть, величины $t_{1}=\tau_{1} /\left(\lambda_{1}+\tau_{1}\right), \ldots, t_{d}=\tau_{d} /\left(\lambda_{d}+\tau_{d}\right)$ всегда будут удовлетворять соотношениям (7).

Отметим, что если выполнено равенство (3), то симплекс $\mathfrak{B}$ вырождается в точку и величины $t_{j}$ можно выбрать только единственным образом. Последнее наблюдение послужило поводом для формулировки замечания 3 в настоящей работе.

\section{СПИСОК ЦИТИРОВАННОЙ ЛИТЕРАТУРЫ}

1. Newhouse S. // Proc. Sympos. Pure Math. 1970. V. 14. P. 191-203. 2. Newhouse S. // Topology. 1974. V. 13. P. 9-18. 3. Newhouse S. // IHES Publ. Math. 1983. V. 50. P. 433-459. 4. Hall M., Jr. // Ann. of Math. (2). 1948. V. 48. P. 966-993. 5. Мощевитин Н. Г. // УМН. 1997. T. 52. №6. C. 145-146. 6. Williams R. F. // Contemp. Math. 1991. V. 117. P. 163-175. 7. Hlavka J. L. // Trans. Amer. Math. Soc. 1975. V. 211. P. 123-134. 8. Astels S. // Electronic Research Announcements of the Amer. Math. Soc. 1999. V. 5. P. 108-111; // Trans. Amer. Math. Soc. 2000. V. 352. №1. P. 133-170. 9. Cusick T. W. // Proc. Amer. Math. Soc. 1973. V. 38. № 2. P. 253-254. 10. Cusick T. W., Lee R. A. // Proc. Amer. Math. Soc. 1971. V. 30. № 2. P. $241-248$. 11. Cusick T. W., Flachive M. E. The Markoff and Lagrange Spectra. Math. Surveys Monographs. V. 30. Providence, R.I.: Amer. Math. Soc., 1989. 12. Shallit J. // L'Enseignment Mathématique. 1992. V. 38. Р. 151-187. 13. Мощевитин Н. Г. // УМН. 1999. Т. 54. № 3. С. 169-170.

Московский государственный университет им. М.В.Ломоносова 